\title{
The Magnetic Corona: Magnetic Reconnection in Solar Flares
}

\author{
Harry P. Warren \\ E. O. Hulburt Center for Space Research, Code 7670, Naval Research \\ Laboratory, Washington, DC 20375, U.S.A.
}

\begin{abstract}
The ability of the Transition Region and Coronal Explorer (TRACE) to image the Sun at high spatial resolution and high cadence over a very broad range of temperatures makes it a unique instrument for observing solar flare plasma. TRACE observations have confirmed the reconnection model for solar flares, at least qualitatively. TRACE flare observations show impulsive footpoint brightenings that are followed by the formation of high-temperature loops in the corona. These loops then cool to lower temperatures, forming post-flare loop arcades. Comparisons between TRACE and lower spatial resolution Yohkoh Soft X-Ray Telescope (SXT) observations have revealed that solar flares are composed of a multitude of fine coronal loops. Detailed hydrodynamic modeling of flare light curves shows that this fine scale structuring is crucial to understanding the evolution of the observed emission. Models based on single, isothermal loops are not consistent with the TRACE observations. Models based on the sequential heating of small-scale loops, in contrast, are able to reproduce many of the salient features of the observed light curves. We will discuss the implication of these results for more energetic stellar flares as well as smaller-scale events that may be responsible for the heating of solar active region loops.
\end{abstract}

\section{Introduction}

Despite many years of observational and theoretical effort a detailed understanding of how the solar corona is heated to high temperatures remains elusive. For example, it is now widely believed that magnetic reconnection is the primary source of energy for driving large solar flares (see, for example, Forbes \& Acton 1996). Much of the evidence for the role of magnetic reconnection, such as the observation of cusp-shaped loops (e.g., Tsuneta et al. 1992) or the detection of coronal hard X-ray sources (e.g., Masuda et al. 1994) remains indirect, however. There are also indications of problems with this model. Hydrodynamic simulations, for example, indicate that very high velocities $\left(\sim 500 \mathrm{~km} \mathrm{~s}^{-1}\right)$ should be observed during the rise phase of a solar flare (Mariska, Emslie, \& Li 1989). Observations, however, show that high temperature line profiles in solar flares are almost always dominated by a stationary component (Mariska, Doschek, \& Bentley 1993).

The Transition Region and Coronal Explorer (TRACE), a normal incidence, multi-layer solar telescope which was launched in 1998 as part of NASA's Small 
Explorer program, has three capabilities that make it a unique instrument for solar flare observations. First, TRACE has a spatial resolution of $1^{\prime \prime}\left(0^{\prime \prime} 5\right.$ pixels). This is higher than the nominal resolution of $5^{\prime \prime}$ achieved with previous grazing incidence telescopes used to study solar flares, such as the Yohkoh Soft X-Ray Telescope (SXT, Tsuneta et al. 1991). Second, TRACE can image plasma over a very wide range of temperatures $\left(10^{4}-10^{7} \mathrm{~K}\right)$. The sensitivity of TRACE to high temperature plasma is derived from the presence of the CaXVII $193 \AA$ and Fe XXIV $192 \AA$ flare lines in its $195 \AA$ channel in addition to Fe XII $195 \AA$. Thus TRACE can observe the pre-flare corona, the response of the chromosphere and transition region to energy deposition, the formation of high temperature flare loops in the corona, and the cooling of post-flare loop arcades. Finally, TRACE can observe at cadences as high as about $2 \mathrm{~s}$, depending on the wavelength and other observational parameters.

TRACE flare observations have confirmed the essential elements of models based on magnetic reconnection, at least at a qualitative level. TRACE observations of two-ribbon flares (e.g., Warren et al. 1999; Aschwanden \& Alexander 2001) show impulsive footpoint brightenings that are quickly followed by the appearance of an arcade of hot $(\sim 20 \mathrm{MK})$ flare loops. As hot loops are formed at progressively larger heights, cooler $(\sim 1 \mathrm{MK})$ loops begin to form at the lowest heights. The delay between the formation of the hot flare loops and the cooler post-flare loops is approximately consistent with cooling by conduction and radiation. These results are generally consistent with previous observations with other instruments (e.g., Švestka 1982; Harra-Murnion et al. 1998).

The broad temperature coverage and high spatial resolution of TRACE have revealed that that there is considerably more fine scale structure in solar flares than was previously imagined. The evidence for filamentation is both direct and indirect. The high spatial resolution TRACE observations clearly show the formation of small-scale, post-flare loops (e.g., Warren 2000). The detailed quantitative analysis of TRACE and SXT flare light curves also indicates that the formation of a succession of small-scale loops is essential for reproducing the observed emission (Warren \& Reeves 2001; Reeves \& Warren 2002). In this paper we will review the evidence for filamentation in solar flares and discuss its relevance to the modeling of stellar flares. Recent work suggested that impulsive heating and filamentation may also be important in explaining the properties of active region loops observed with TRACE (e.g., Warren, Winebarger, \& Mariska 2003) and we will also briefly discuss these results. Taken together, the TRACE observations of flares and active region loops provide strong evidence for a highly dynamic and highly filamented solar corona. The filamentary nature of the energy release process suggests that magnetic reconnection plays a significant role in heating the corona.

\section{Instrumentation}

For completeness, a few details on the TRACE and SXT instruments are provided here. The TRACE instrument is described in detail by Handy (1999). The initial in-flight performance is reviewed by Golub et al. (1999) and Schrijver et al. (1999). SXT is described in detail by Tsuneta et al. (1991). 
TRACE is based on a $30 \mathrm{~cm}$ Cassegrain telescope. The primary and secondary mirrors are divided into quadrants and a rotating shutter is used to select which quadrant is illuminated. Only a single quadrant can be used at a time. Images in all of the wavelengths are projected onto a single detector, a $1024 \times 1024$ CCD. Each CCD pixel represents a solar area about 0.'5 on a side.

Three of the mirror quadrants are coated with multilayers for imaging at extreme ultraviolet (EUV) wavelengths. The multilayer coatings have peak sensitivities at approximately $171 \AA, 195 \AA$, and $284 \AA$, and widths of $7-10 \AA$. The $171 \AA$ bandpass is typically dominated by the Fe IX $171 \AA$ and Fe X $174 \AA$ emission lines. Under most conditions the $195 \AA$ bandpass is dominated by the Fe XII $195 \AA$ emission line. During active region observations the primary contribution to the $284 \AA$ bandpass is from Fe XV $284 \AA$. During flares thermal bremsstrahlung makes a contribution to all three channels (Feldman et al. 1999). This contribution is weak, however, compared with the emission from the background corona, and can only be observed in the largest flares. As mentioned previously, the $195 \AA$ channel is sensitive to flare plasma because of the CaXVII $193 \AA$ and Fe XXIV $192 \AA$ flare lines in this wavelength range.

The fourth mirror quadrant is coated with aluminum and magnesium fluoride. This channel, in combination with focal plane filters, is used for imaging very broad wavelength ranges near $1216 \AA, 1550 \AA, 1600 \AA$, and $1700 \AA$. For most quiet Sun and active region observations these bandpasses are dominated by cool chromospheric continuum and line emission. During flares there is some evidence that transition region emission lines such as C IV $1548 \AA$ and $1550 \AA$ may brighten by as much as a factor of $10^{4}$ while the cooler emission only increases in intensity by a factor of about $10^{2}$ (Brekke et al. 1996). Thus during intense solar flares the 1550 and $1600 \AA$ channels can be dominated by transition region emission.

The SXT is one of four instruments flown on the Yohkoh spacecraft, which operated between October 1991 and December 2001. The SXT is a grazing incidence telescope that is sensitive to emission between about 3 and $45 \AA$. Temperature discrimination is achieved through the use of several focal plane filters. The thickest filters are the "thick aluminium" (Al12) filter, which is $12 \mu \mathrm{m}$ of $\mathrm{Al}$, and the "beryllium" (Be119) filter, which is $119 \mu \mathrm{m}$ of Be. Images in the thinner filters, which allow SXT to observe plasma down to a temperature of about $2 \mathrm{MK}$, saturate during most large flares. Thus SXT generally can observe only high temperature plasma during solar flares. The spatial resolution of SXT is about $5^{\prime \prime}(2$ "' 46 pixels).

\section{Solar and Stellar Flares}

Perhaps the simplest way to illustrate the similarities and differences between TRACE and SXT flare observations is to consider some nearly simultaneous images. Shown in Figure 1 are selected images from the June 22, 1999 M1.7 tworibbon flare that began at about 18:10 UT, peaked at 18:29 UT, and decayed over about an hour. All of the TRACE and SXT images taken near the peak of the hard X-ray flux show intense footpoint brightenings. As the flare progresses, the TRACE $195 \AA$ and SXT images then show the formation of an arcade of high temperature loops. Lacking a significant response at flare temperatures the 

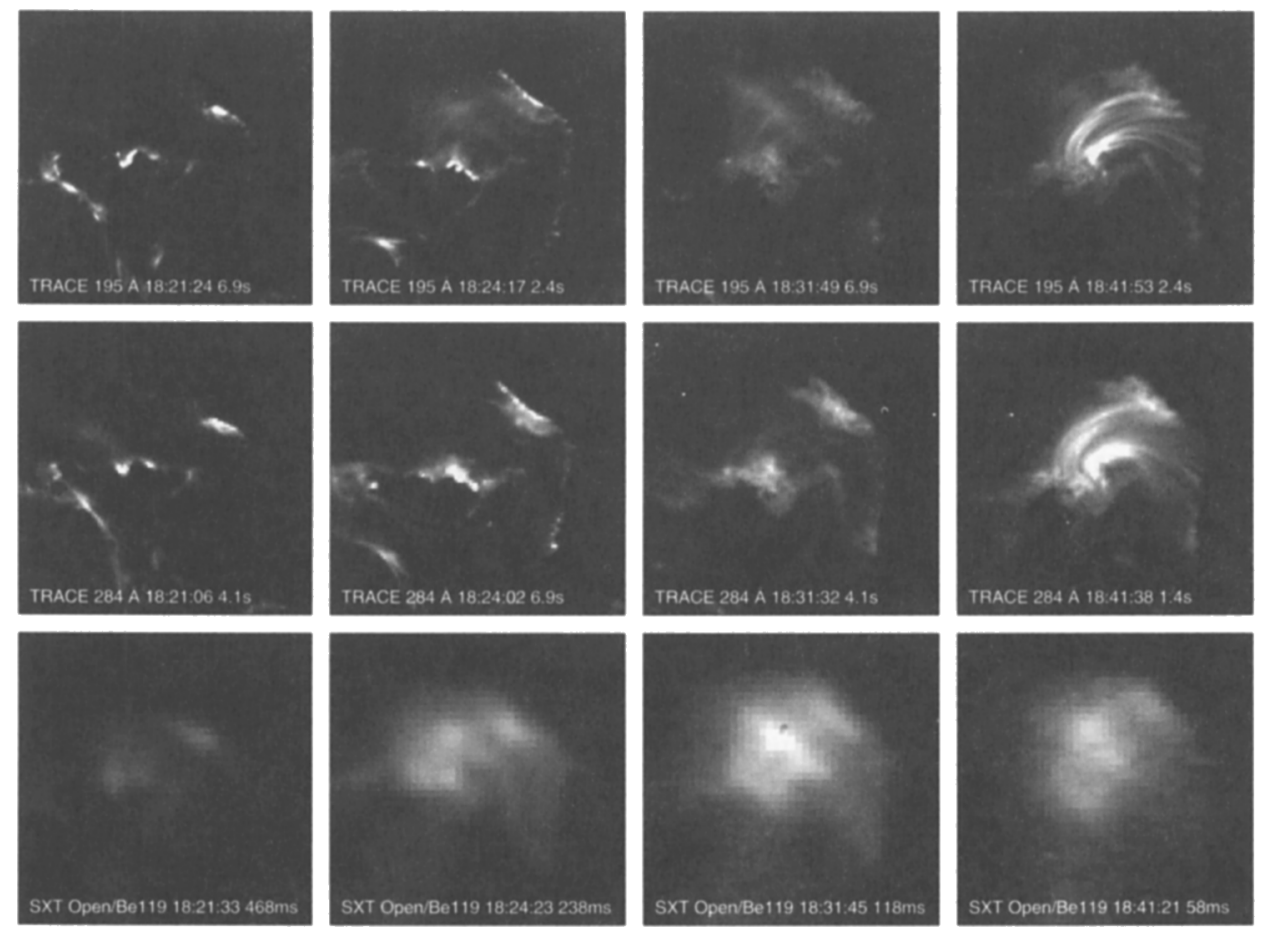

Figure 1. TRACE and SXT images from the 1999 June 22 M1.7 flare which began at about 18:20 and peaked at about 18:30. The images cover an area of $100^{\prime \prime} \times 100^{\prime \prime}$ and are scaled logarithmically. The high temperature Fe XXIV flare plasma in the TRACE $195 \AA$ channel can be differentiated from the cooler Fe XII emission through comparisons with the TRACE $284 \AA$ images. After Warren (2000).

TRACE $171 \AA$ and $284 \AA$ channels show only footpoint emission. It is not until about 18:34, approximately 15 minutes after the peak in the soft X-ray fluxes, that loops begin to appear in the TRACE $171 \AA$ and $284 \AA$ images. These cooler post-flare loops are also evident in the TRACE $195 \AA$ images.

The observations from this flare confirm the ordering of events given by the magnetic reconnection theory. This event shows impulsive footpoint brightenings followed by the formation of high temperature loops and the subsequent cooling of these loops to cooler temperatures. These images also clearly illustrate the significant improvement in spatial resolution offered by the normal incidence design of TRACE. The high resolution TRACE images show that the flare plasma is not confined to a single loop or even a few isolated loops, but a multitude of fine coronal structures.

One unexpected aspect of these TRACE observations are the apparent differences in morphology between the high temperature flare plasma and the cooler post-flare emission. The high temperature plasma imaged in the TRACE $195 \AA$ channel appears diffuse while the cooler arcade that forms later in the event 
appears decidedly loop-like. As we will see, this difference does not reflect fundamental differences in plasma confinement at different temperatures. Instead, these differences reflect the differences in the cooling at different temperatures.

To investigate the evolution of flare plasma as it cools through the SXT and TRACE bandpasses Reeves \& Warren (2002) have utilized the "point" loop model of Cargill, Mariska, \& Antiochos (1995). In this model only the temporal evolution of the temperature and density are calculated, the structure along the loop is not considered. This treatment is predicated on the assumption that the cooling is dominated either by conductive cooling or radiative cooling. This permits the hydrodynamic loop equations to be solved using the separation of variables. Under this assumption the temporal evolution of the temperature is of the form

$$
T_{e}(t)=T_{e}(t=0)\left[1-\frac{t}{\tau}\right]^{\beta},
$$

where $\tau$ is related to either the conductive or radiative time scale and $\beta$ is a constant. The timescale for conductive cooling is

$$
\tau_{c}=4 \times 10^{-10} \frac{n_{e} L^{2}}{T_{e}^{5 / 2}},
$$

where $T_{e}$ and $n_{e}$ are the electron temperature and density, and $L$ is the half length of the loop. The radiative timescale is

$$
\tau_{r}=\frac{3 k_{B} T_{e}^{1-\alpha}}{n_{e} \chi}
$$

where $\alpha$ and $\chi$ are determined from the radiative loss function, $P_{r d}=\chi T_{e}^{\alpha}$, and $k_{B}$ is the Boltzman constant.

Additional assumptions are needed to address the evolution of the density. In the Cargill et al. (1995) model it is assumed that the pressure is constant during the conductive phase. Thus during the conductive phase the density rises as the temperature falls. During the radiative phase the $n_{e} \propto \sqrt{T_{e}}$ scaling law suggested by Serio et al. (1991) and Jakimiec et al. (1992) from full hydrodynamic simulations of solar flares is used.

To simulate the evolution of a loop an initial temperature and density are assumed. These values are used to calculate the conductive and radiative timescales. The smaller of the two timescales is used to evolve the equation for the temperature (Equation 1). The updated value for the temperature is then used to advance the density. This process is repeated to determine the evolution of the loop. The advantage of the Cargill et al. (1995) point loop model over full hydrodynamic simulations is that it allows for the rapid calculation of solutions for many loops.

It is possible to simulate a post-flare loop arcade by considering a series of progressively longer loops that "turn on" at successively later times. An example of such a calculation is shown in Figure 2, where densities and temperatures calculated using the Cargill et al. (1995) model have been used to simulate the emission in the TRACE $195 \AA$ channel. This simulation reproduces several of important features of the observations. The emission during the peak of the flare, when the temperature is about $20 \mathrm{MK}$, does not show well defined loops. 
As the plasma cools through 1.5 MK, however, the emission becomes loop-like. The radiative losses are substantial as the plasma cools through the TRACE bandpasses, so the loop temperature evolves rapidly at these temperatures. Furthermore, the emissivities of the TRACE EUV bandpasses are sharply peaked functions of temperature, thus bright emission is seen in TRACE bandpasses for only a short time. Finally, the loop-like emission in the arcade appears to
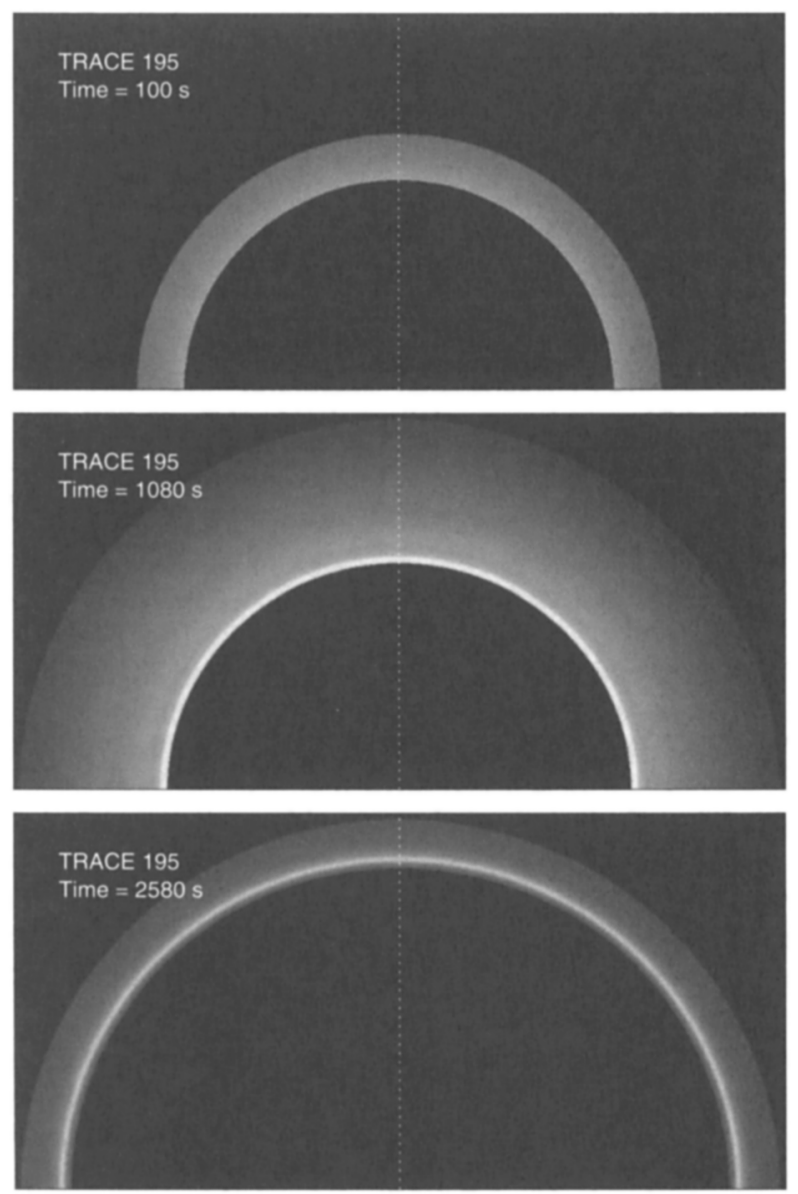

Figure 2. Snapshots from different times in a flare cooling simulation, passed through the TRACE 195 passband. The top frame is early in the flare, when most of the emission is due to the high-temperature Fe XXIV at $192 \AA$ that is detected by the TRACE $195 \AA$ passband. The middle frame shows the formation of the bright loop structure due to Fe XII at $1.5 \mathrm{MK}$, which is the most significant contribution to the 195 $\AA$ bandpass. The bottom frame shows a late time in the flare, when high, previously hot loops have cooled to $1.5 \mathrm{MK}$. The emission has been assumed to be uniform along the loop. From Reeves \& Warren (2002). 

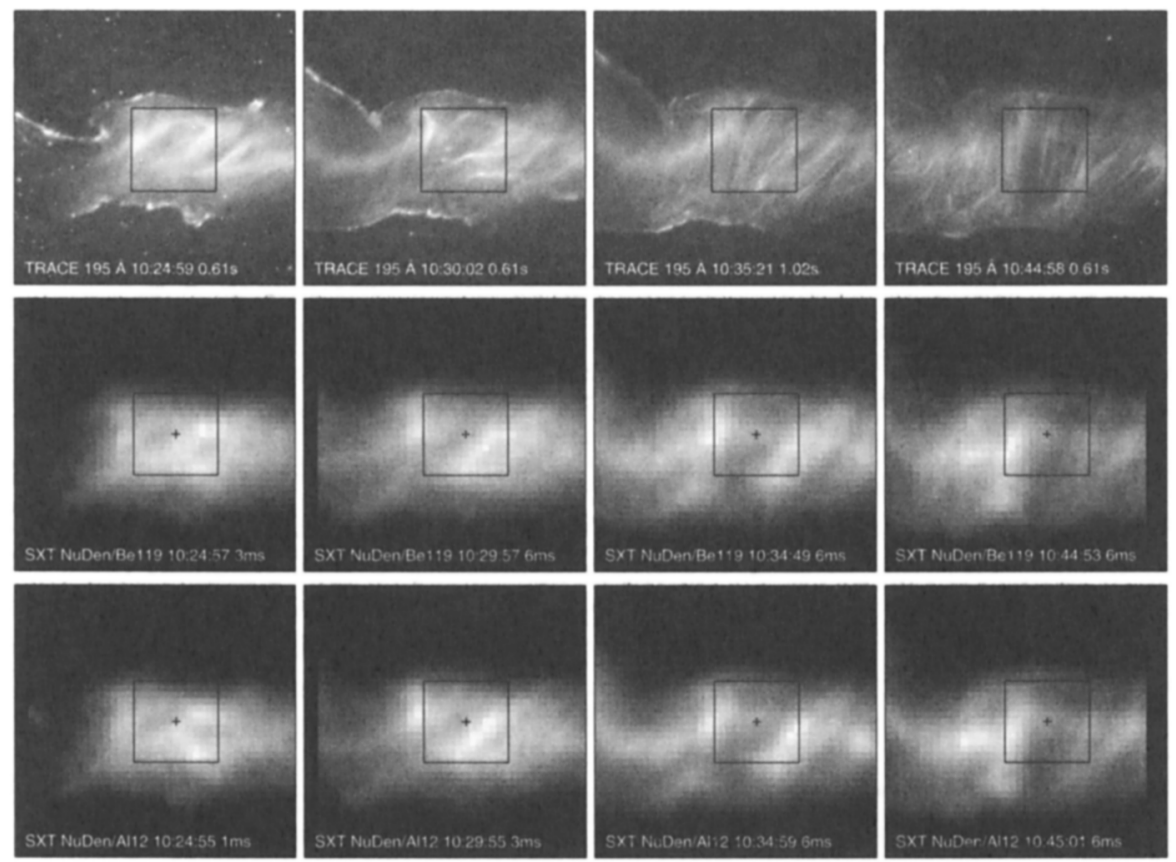

Figure 3. TRACE $195 \AA$ and SXT Be119 and Al12 images from the July 14, $2000 \mathrm{X} 6$ flare. The black box on the images indicates the area used to calculate light curves. From Reeves \& Warren (2002).

rise with time as loops at progressively larger heights cools through the TRACE bandpasses.

Reeves \& Warren (2002) compared the predictions of this multi-loop model with TRACE and SXT observations of the July 14, 2000 X6 flare. Images from this two-ribbon flare, as shown in Figure 3, are similar in morphology and evolution to the June 22, 1999 flare discussed earlier. This flare began at about 10:05 UT, peaked at 10:24, and decayed over several hours.

To simulate the evolution of this flare arcade the ribbon spreading velocity is used to determine the rate at which loops are activated. Simulated and observed light curves are shown in Figure 4. For comparison, both a singleloop simulation and multi-loop simulation are displayed. A significant feature of the single loop simulation, which is clearly not present in the observations, is the large spike in the TRACE $195 \AA$ flux as all of the plasma in the loop cools through $1.5 \mathrm{MK}$ simultaneously. We also see that the single loop model generally does a poor job of reproducing the decay of emission in all three bandpasses. The agreement between the simulated and observed SXT light curves could be improved significantly if there was some extended heating in the loop. Since all of the plasma would cool through $1.5 \mathrm{MK}$ at some point, extended heating would not remove the large spike in the simulated TRACE $195 \AA$ emission. Because only a small fraction of the arcade is passing through $1.5 \mathrm{MK}$ at a given time in the multi-loop model, it does not show a large spike in the calculated TRACE 

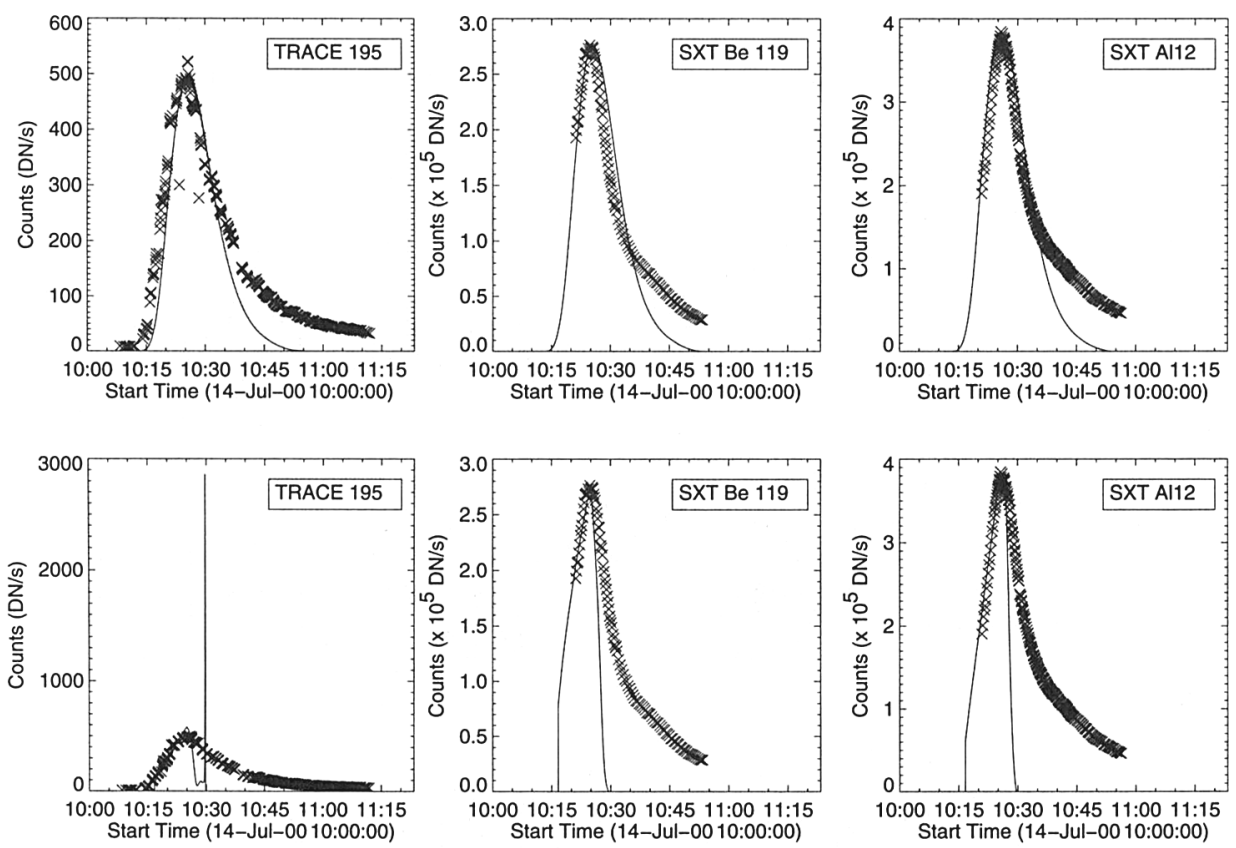

Figure 4. Observed and modeled light curves for the July 14, 2000 $\mathrm{X} 6$ flare. (top panels) Light curves derived from a single loop model of the flare. Note the large spike in the TRACE $195 \AA$ bandpass as all of the flare plasma passes through $1.5 \mathrm{MK}$ simultaneously. (bottom panels) Light curves derived from a multi-thread model of the flare. From Reeves \& Warren (2002).

$195 \AA$ light curve. The multi-loop model also is in somewhat better agreement with the observations during the decay, although there are still some significant discrepancies. These discrepancies are likely due to the loop geometry assumed in the model. Toward the end of the event the ribbons become faint and it is difficult to measure the ribbon spreading velocity accurately. Also, the loops become progressively more dipolar but semi-circular loops have been assumed.

The distinction between single loop and multi-loop modeling is relevant to the interpretation of stellar flare. Stellar flares have been observed that have significantly higher peak temperatures and emission measures than even the largest solar flares. The quasi-static modeling of these events (e.g., van den Oord \& Mewe 1989) have suggested very large loop lengths (comparable to a stellar radius). Other work which assumes sustained heating of the flare loop (e.g., Favata, Micela, \& Reale 2001) indicates lengths that are much shorter than a stellar radius, similar to what is observed in solar flares. The multiloop modeling of solar flares, however, suggests that these approaches are not correct. Solar flares are generally not composed of single loops and sustained heating does not appear to be needed to reproduce the observed fluxes in solar 
flares. There is a clear need to apply this multi-loop modeling to stellar flare observations.

\section{Active Region Loops}

Recent work by Lenz et al. (1999) and Aschwanden, Nightingale, \& Alexander (2000) has shown that many active region loops observed with TRACE are not consistent with the Rosner, Tucker, Vaiana, Serio (RTVS) scaling laws for static, uniformly heated loops (Rosner, Tucker, \& Vaiana 1978; Serio et al. 1981). The observations show loops that have much larger intensities than can be accounted for by the scaling laws for uniform heating. That is, the observed loops are "overdense" relative to the model predictions. The scaling laws also predict significant temperature gradients along the loops, while the observations show nearly constant TRACE $195 \AA / 171 \AA$ filter ratios. The observations also show loops that persist for much longer than a characteristic cooling time. Static loop models with heating localized at the footpoints generally have flatter temperature profiles and larger apex densities than uniformly heated loops, but the increase in density is generally not large enough to reproduce the observed intensities (Winebarger, Warren, \& Mariska 2003; Aschwanden, Schrijver, \& Alexander 2001).

Recent work by Warren, Winebarger, \& Hamilton (2002) and Spadaro et al. (2003) has emphasized the role of impulsive heating in explaining the observed properties of these loops. Hydrodynamic simulations of large solar flares have shown that the density in a cooling, post-flare loop generally declines much more slowly than the temperature. Serio et al. (1991) and Jakimiec et al. (1992), for example, argue for a $n_{e} \propto \sqrt{T_{e}}$ relationship between the density and the temperature as the loop cools. This scaling law indicates that the density in cooling post-flare loop would drop only by a factor of about 3 as the temperature declines from $10 \mathrm{MK}$ to $1 \mathrm{MK}$. In contrast, the RTVS scaling laws indicate a $n_{e} \propto T_{e}^{2}$ relationship between the apex density and temperature. A decline in temperature between $10 \mathrm{MK}$ and $1 \mathrm{MK}$ would require the density to drop by a factor of about 100 for the loop to conform to the RTVS scaling laws. Thus it is possible for a post-flare loop to be far from hydrostatic equilibrium as it cools through $1 \mathrm{MK}$.

Winebarger, Warren, \& Seaton (2003) analyzed the temporal evolution of 5 relatively isolated active region loops observed with $T R A C E$ and found that all of these loops appeared first in the hotter $195 \AA$ filter and then in the cooler $171 \AA$ filter, which is qualitatively consistent with impulsively heated loops cooling through the TRACE bandpasses. Data for the shortest loop they analyzed is shown in Figure 5. This loop was also part of the Aschwanden et al. (2000) study. For this loop, the light curves indicate a delay of $150 \pm 30 \mathrm{~s}$ between the appearance of the loop in the $195 \AA$ and $171 \AA$ channels.

Warren, Winebarger, \& Mariska (2003) were able to reproduce the temporal and spatial evolution of this loop by modeling it as a bundle of small-scale, impulsively heated filaments. The filamentation was necessary to reproduce the flat filter ratios and the extended lifetime of the observed emission. A single, simulated loop that exhibits the observed delay fades away much too quickly. Also, the simulations indicate that a single impulsively heated loop has a sig- 

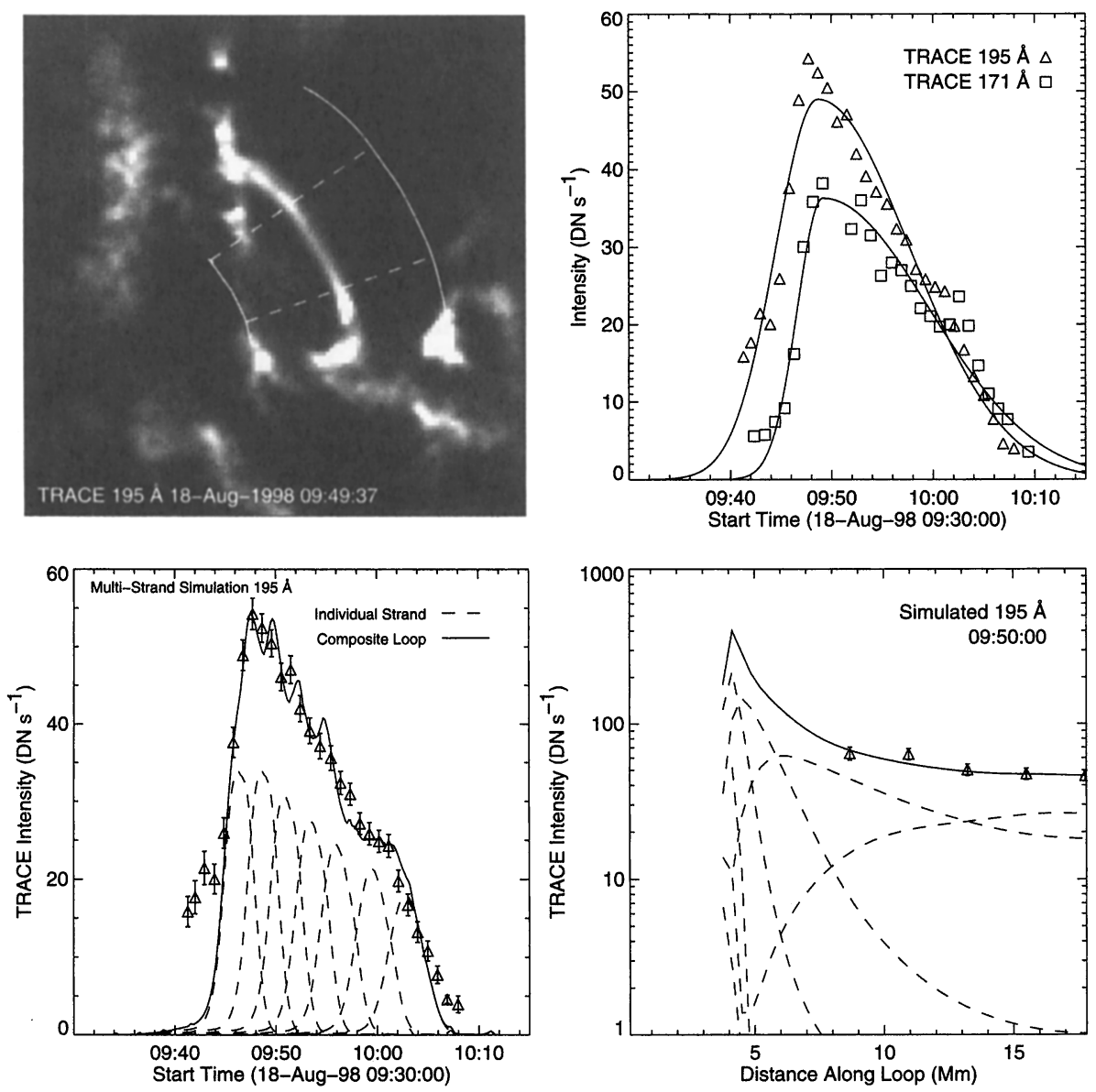

Figure 5. Observation and modeling of an active region loop. (top left) A TRACE $195 \AA$ image from near the peak intensities. The lines indicate the region used to determine the background subtracted intensities as a function of time. (top right) TRACE 171 and $195 \AA$ intensities as a function of time. The solid lines are asymmetric Gaussian fits that are used to determine the delay. (bottom left) Simulated intensities as a function of time derived from a multi-thread model. (bottom right) Simulated intensities as a funtion of position along the loop. After Warren, Winebarger, \& Mariska (2003).

nificant temperature gradient along its length. As shown in Figure 5, modeling the loop as a succession of impulsively heated filaments generally reproduces the high densities, the observed delay, the extended lifetime, and the distribution of intensity along the loop. 


\section{Discussion}

TRACE's combination of high spatial resolution, broad temperature coverage, and high cadence has greatly advanced our understanding of the morphology and evolution of solar flares. Both simple inspection and detailed modeling of the TRACE flare observations clearly show that flares must be composed of many small-scale loops. Furthermore, the successful application of this multiloop modeling to active region loop observations suggests that the energy release process in flare may be relevant to more general problem of the heating the solar corona. In both cases the heating of a succession of small-scall loops is needed to reproduce the observations. This points to magnetic reconnection as playing an important role in coronal heating. Of course, only a few examples have been considered. Much more work needs to be done to determine how general these results are.

These results also have important implications for future solar missions. Instruments with high spatial, spectral, and temporal resolution are needed to fully explore the highly filamented and highly dynamic solar corona hinted at by the TRACE observations.

Acknowledgments. TRACE is supported by a NASA contract to the Lockheed Martin Advanced Technology Center. Yohkoh is a mission of the Institute of Space and Astronautical Sciences (Japan), with participation from the U.S. and U.K. This work has been supported by the NASA Sun-Earth Connection Guest Investigator Program, The NASA Supporting Research and Technology Program, and by the Office of Naval Research.

\section{References}

Aschwanden, M. J., Nightingale, R. W., \& Alexander, D. 2000, ApJ, 541, 1059 Aschwanden, M. J., \& Alexander, D. 2001, Solar Phys., 204, 91

Aschwanden, M. J., Schrijver, C. J., \& Alexander, D. 2001, ApJ, 550, 1036

Brekke, P., Rottman, G. J., Fontenla, J., \& Judge, P. G. 1996, ApJ, 468, 418

Cargill, P. J., Mariska, J. T., Antiochos, S. K. 1995, ApJ, 439, 1034

Favata, F., Micela, G., Reale, F. 2001, A\&A, 375, 485

Feldman, U., Laming, J. M., Doschek, G. A., Warren, H. P., Golub, L. 1999, ApJ, 511, L61

Forbes, T. G., \& Acton, L. W. 1996, ApJ, 459, 330

Golub, L., Bookbinder, J., Deluca, E., Karovska, M., Warren, H., Schrijver, C. J., Shine, R., Tarbell, T., Title, A., Wolfson, J., Handy, B., Kankelborg, C. 1999, Phys. Plasmas, 6, 2205

Handy, B. N., Acton, L. W., Kankelborg, C. C., Wolfson, C. J., Akin, D. J., Bruner, M. E., Caravalho, R., Catura, R. C., Chevalier, R., Duncan, D. W., Edwards, C. G., Feinstein, C. N., Freeland, S. L., Friedlaender, F. M., Hoffmann, C. H., Hurlburt, N. E., Jurcevich, B. K., Katz, N. L., Kelly, G. A., Lemen, J. R., Levay, M., Lindgren, R. W., Mathur, D. P., Meyer, S. B., Morrison, S. J., Morrison, M. D., Nightingale, R. W., Pope, T. P., Rehse, R. A., Schrijver, C. J., Shine, R. A., Shing, L., Strong, K. T., 
Tarbell, T. D., Title, A. M., Torgerson, D. D., Golub, L., Bookbinder, J. A., Caldwell, D., Cheimets, P. N., Davis, W. N., Deluca, E. E., McMullen, R. A., Warren, H. P., Amato, D., Fisher, R., Maldonado, H., \& Parkinson, C. 1999, Solar Phys., 187, 229

Harra-Murnion, L. K., Schmieder, B., van Driel-Gesztelyi, L., Sato, J., Plunkett, S. P., Rudawy, P., Rompolt, B., Akioka, M., Sakao, T., \& Ichimoto, K. 1998, Solar Phys., 337, 911

Jakimiec, J., Sylwester, B., Sylwester, J., Serio, S., Peres, G., Reale, F. 1992, 253,269

Lenz, D. D., Deluca, E. E., Golub, L., Rosner, R., \& Bookbinder, J. A. 1999, ApJ, 517, L155

Mariska, J. T., Emslie, A. G., \& Li, P. 1989, ApJ, 341, 1067

Mariska, J. T., Doschek, G. A., \& Bentley, R. D. 1993, ApJ, 419, 418

Masuda, S., Kosugi, T., Hara, H., Tsuneta, S., \& Ogawara, Y. 1994, Nature, 371,495

Reeves, K. K., \& Warren, H. P. 2002, ApJ, 578, 590

Rosner, R., Tucker, W. H., \& Vaiana, G. S., 1978, ApJ, 220, 643

Serio S., Peres, G., Vaiana, G. S., Golub, L., \& Rosner, R. 1981, ApJ, 243, 288

Serio, S., Reale, F., Jakimiec, J., Sylwester, B., Sylwester, J. 1991, 241, 197

Schrijver, C. J., Title, A. M., Berger, T. E., Fletcher, L., Hurlburt, N. E., Nightingale, R. W., Shine, R. A., Tarbell, T. D., Wolfson, J., Golub, L., Bookbinder, J. A., Deluca, E. E., McMullen, R. A., Warren, H. P., Kankelborg, C. C., Handy, B. N., \& de Pontieu, B. 1999, Solar Phys., 187,261

Spadaro, D., Lanza, A. F., Lanzafame, A. C., Karpen, J. T., Antiochos, S. K., Klimchuk, J. A., \& MacNeice, P. J. 2003, ApJ, 582, 486

Švestka, Z., Dodson-Prince, H. W., Mohler, O. C., Martin, S. F., Moore, R. L., Nolte, J. T., \& Petrasso, R. D. 1982, Solar Phys., 271

Tsuneta, S., Acton, L., Bruner, M., Lemen, J., Brown, W., Caravalho, R., Catura, R., Freeland, S., Jurcevich, B. \& Owens, J. 1991, Solar Phys., 136,37

Tsuneta, S., Hara, H., Shimizu, T., Acton, L. W., Strong, K. T., Hudson, H. S., \& Ogawara, Y. 1992, PASJ, 44, L63.

van den Oord, G. H. J., \& Mewe, R. 1989, A\&A, 213, 245

Warren, H. P., Bookbinder, J. A., Forbes, T. G., Golub, L., Hudson, H. S., Reeves, K., \& Warshall, A. 1999, ApJ, 527, L121

Warren, H. P. 2000, ApJ, 536, L105

Warren, H. P., \& Reeves, K. K., 2001, ApJ, 554, L103

Warren, H. P., Winebarger, A. R., Mariska, J.T. 2003, ApJ, 593, 1174

Warren, H. P., Winebarger, A. R., \& Hamilton, P. S. 2002, ApJ, 579, L41

Winebarger, A. R., Warren, H. P., \& Mariska, J. T. 2003, ApJ, 587, 439

Winebarger, A. R., Warren, H. P., \& Seaton, D. B, 2003, ApJ, 593, 1164 